\title{
Unheard voices: outcomes of tertiary care for treatment-refractory psychosis
}

\author{
S. Neil Sarkar, ${ }^{\star 1,2}$ Derek K. Tracy, ${ }^{\star 2,3}$ Maria-Jesus Mateos Fernandez, ${ }^{2}$ Natasza Nalesnik, ${ }^{2}$ \\ Gurbinder Dhillon, ${ }^{3}$ Juliana Onwumere, ${ }^{4}$ Anne-Marye Prins, ${ }^{4}$ Karen Schepman, ${ }^{2}$ Tracy Collier, ${ }^{2}$ \\ Thomas P. White, ${ }^{2}$ Anita Patel, ${ }^{5}$ Fiona Gaughran, ${ }^{4}$ Sukhwinder S. Shergill ${ }^{2,4}$
}

Psychiatric Bulletin (2014), 38, 71-74, doi: 10.1192/pb.bp.112.042598

\begin{abstract}
${ }^{1}$ Central and North West London NHS Foundation Trust; ${ }^{2}$ Cognition,

Schizophrenia and Imaging Laboratory, Department of Psychosis Studies, Institute of Psychiatry, King's College London; ${ }^{3}$ Oxleas NHS Foundation Trust, London; ${ }^{4}$ South London and Maudsley NHS Foundation Trust; ${ }^{5}$ Health Service and Population Research Department, Institute of Psychiatry, King's College London First received 31 Dec 2012, final revision 10 Jul 2013, accepted 12 Jul 2013

Correspondence to Sukhwinder S Shergill (sukhi.shergill@kcl.ac.uk)

(C) 2014 The Royal College of

Psychiatrists. This is an open-access article published by the Royal College of Psychiatrists and distributed under the terms of the Creative Commons Attribution License (http:// creativecommons.org/licenses/by/ 3.0), which permits unrestricted use, distribution, and reproduction in any medium, provided the original work is properly cited.
\end{abstract}

Aims and method In up to a quarter of patients, schizophrenia is resistant to standard treatments. We undertook a naturalistic study of 153 patients treated in the tertiary referral in-patient unit of the National Psychosis Service based at the Maudsley Hospital in London. A retrospective analysis of symptoms on admission and discharge was undertaken using the OPCRIT tool, along with preliminary economic modelling of potential costs related to changes in accommodation.

Results In-patient treatment demonstrated statistically significant improvements in all symptom categories in patients already identified as having schizophrenia refractory to standard secondary care. The preliminary cost analysis showed net savings to referring authorities due to changes from pre- to post-discharge accommodation.

Clinical implications Despite the enormous clinical, personal and societal burden of refractory psychotic illnesses, there is insufficient information on the outcomes of specialised tertiary-level care. Our pilot data support its utility in all domains measured.

Declaration of interest S.N.S., D.K.T., M.-J.M.F., G.D., J.O., A.-M.P., K.S., F.G. and S.S.S. work, or have worked, on the National Psychosis Unit where the study was undertaken.
In about a quarter of patients with schizophrenia who adhere to treatment, the illness proves refractory to standard treatment protocols. ${ }^{1,2}$ Up to $50 \%$ of patients do not respond to clozapine, and there is a lack of novel pharmacological treatments. ${ }^{3,4}$ Various national and international treatment-refractory psychosis guidelines exist: ${ }^{1,5-7}$ most promote a pragmatic approach to prescribing and patient care. Clinical practice, for various reasons, often deviates from guideline recommendations, and one recent large study ${ }^{8}$ demonstrated an average of five different antipsychotics and a mean delay of 4 years before clozapine is commenced in routine clinical practice.

The National Institute for Health and Care Excellence (NICE) advocates the use of tertiary services for treatmentrefractory illness. The Department of Health has set out guidelines for specialised services in mental health ${ }^{9}$ which acknowledge that 'centres of expertise [which] concentrate skills and experience' are necessary, and offer general

*These authors contributed equally to the work. treatment-refractory and specific refractory psychosis criteria (Box 1). However, there is a lack of outcome data from specialist tertiary service in schizophrenia, despite the prevalence and burden of treatment resistance. This work aimed to explore outcome measures of one such in-patient service: assessing clinical change from admission to discharge, with preliminary modelling of potential costeffectiveness. Although data on tertiary care in psychosis are limited, we predicted that admission to the National Psychosis Unit would result in multidomain clinical improvements and this would prove cost-effective.

\section{Method}

The clinical notes of patients admitted to the National Psychosis Unit between 2001 and 2007 were collated for retrospective analysis using the OPCRIT system. This is a reliable and well-validated tool utilising an electronic checklist of psychopathological symptoms that are scored, with algorithms for clinical diagnosis. ${ }^{10}$ The notes on 
Box 1 Proposed criteria for complex and/or refractory disorder services generally, and psychosis services specifically

\section{Generic complex/refractory criteria}

- Diagnostic uncertainty hampering treatment

- Persistently high symptom burden

- Significant impact on functioning

- Persisting ( $>2$ years) pattern of incapacity despite appropriate treatment

- Multiple comorbidities increasing likelihood of chronicity

- Need for specialised treatments (e.g. transcranial magnetic stimulation)

- In-patient stay $>6-12$ months

Specific to a psychosis centre

- Failure to respond adequately (or tolerate) two antipsychotics (at least one atypical)

- Attempted adequate trial of clozapine, usually for a minimum of 6-9 months

- Appropriate psychological therapies such as cognitivebehavioural therapy and family interventions should have been attempted

Abridged and amended from Department of Health guidelines. ${ }^{9}$

admission to, and discharge from, the National Psychosis Unit were assessed to give comparative OPCRIT scores for each time point. Of 182 sequential notes, 153 had sufficiently detailed clinical information at the time of admission and of discharge for such assessment and accurate completion of OPCRIT scores at the two time points to be made. All patients, 86 male and 67 female, met ICD-10 criteria for a primary diagnosis of schizophrenia, with 36 having a coexistent Axis II diagnosis: 36 had a lifetime history of alcohol dependence or harmful use (24 at the point of admission); 49 a lifetime history of cannabis dependence or harmful use (37 at the point of admission); and 31 a lifetime history of harmful use or dependency on other substances (23 on admission). The mean age on admission was 33 years (s.d. $=10.9$ ), and mean length of stay on the unit was 254 days (s.d. = 169). Thirty patients had their first psychotic episode before the age of 16; in 53 patients it occurred between 17 and 20 years old; in 29, between 21 and 25 years; in 20, between 26 and 35; and 12 had their first episode after the age of 35. At the time of admission, 21 patients were on clozapine monotherapy and 55 patients were taking clozapine with a second antipsychotic.

To evaluate whether there was a significant difference between admission and discharge scores, paired-samples $t$-tests were conducted on the scores for appearance and behaviour, speech and form of thought, affect and associated features, abnormal beliefs and ideas, abnormal perceptions, and total clinical score. Significance was ascribed according to a false-discovery rate (FDR) corrected $P$-threshold of 0.05 having corrected for the number of variables tested.
Sufficient data were available to allow a preliminary analysis of the costs of the pre-admission and postdischarge social care for 96 of the patients admitted. Costs were based on the patient's clinical setting immediately prior to admission $v$. immediately post-discharge, which were categorised as home, residential placement, residential rehabilitation unit, hospital in-patient unit and psychiatric intensive care unit. Costs associated with each setting were estimated by extrapolating baseline data from a previous local study of people with schizophrenia in equivalent settings. ${ }^{11}$ That study obtained costing estimate data from various (UK) sources and involved: social security benefit rates from the UK Department for Work and Pensions; police contact costs; specialist education services costs based on data from the Chartered Institute of Public Finance and Accountancy; medication costs from data in the Joint Formulary Committee; and health and social care service costs based on national estimates - for references see Patel et al. ${ }^{11}$ Costs in this current study were standardised to one-year periods and updated to 2011/2012 prices.

\section{Results}

\section{Clinical outcomes from admission to discharge}

Admission and discharge OPCRIT scores were obtained for all 153 case notes. There was a statistically significant decrease in symptom scores in all domains between admission and discharge (Fig. 1: times 1 and 2 respectively). Significant improvement was seen in: appearance and behaviour $\left(t_{(152)}=7.70, P\right.$-FDR-corrected $\left.=9.8 \times 10-12\right)$; speech and form of thought $\left(t_{(152)}=7.20, P\right.$-FDR-corrected $\left.=1.9 \times 10-10\right)$; affect and associated features $\left(t_{(152)}=11.53, P\right.$-FDR-corrected $=$ $1.0 \times 10-21)$; abnormal beliefs and ideas $\left(t_{(152)}=11.67, P\right.$-FDRcorrected $=4.4 \times 10-22)$; abnormal perceptions $\left(t_{(152)}=8.63\right.$, $P$-FDR-corrected $=4.7 \times 10-14)$; and global clinical score $\left(t_{(152)}=12.72, P\right.$-FDR-corrected $\left.=5.6 \times 10-25\right)$. A breakdown of OPCRIT scores by pre- and post-admission residency is shown in Box 1. Of note, at the time of discharge, 16 of those admitted on clozapine had had their dose increased, and an additional 63 patients had been commenced on this drug.

\section{Cost analysis}

At discharge, the majority of patients moved to the same $(44.8 \%)$ or lower- (also 44.8\%) intensity setting as compared with their pre-admission setting (Table 1). There was an estimated average saving of $£ 20929$ per person per year between pre-admission and post-discharge accommodation costs. The greatest savings were for those who came from the highest-intensity setting, at an estimated average of $£ 41358$ per person, because many of these moved to a lower-intensity setting post-discharge. Those who moved to a lower-intensity setting $(n=43)$ had a higher National Psychosis Unit admission cost (£143493v. $£ 98$ 020; $P$-value from $t$-test 0.009 ) than those who returned to the same or a higher-intensity setting. They also had a greater OPRIT Mental State Examination score improvement (19 v. 9 points; $P=0.002$ ). 


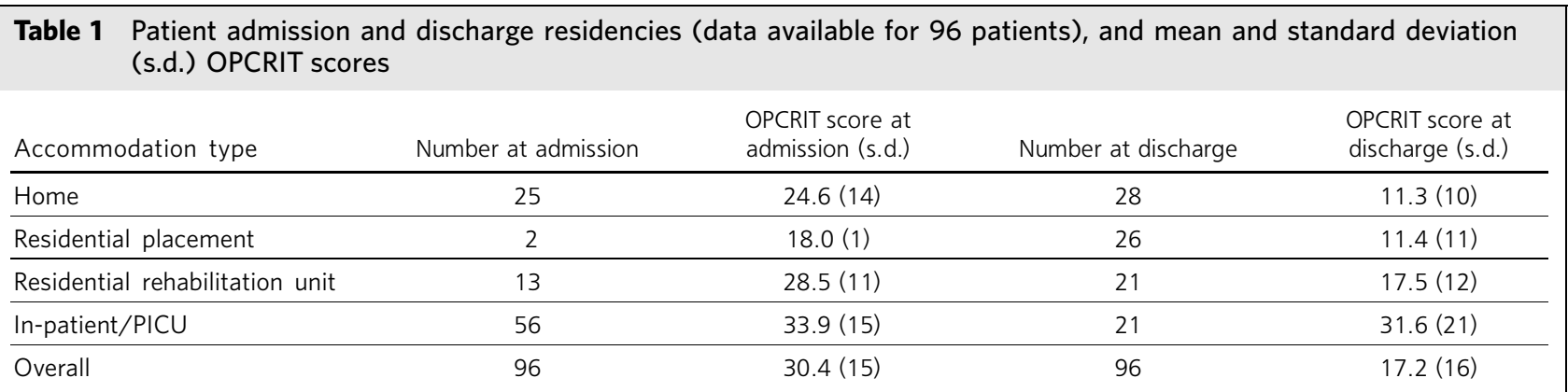

$\mathrm{PICU}$, psychiatric intensive care unit.

\section{Discussion}

Research in refractory psychosis has generally focused on specific individual pharmacological, psychological or sociological interventions, and far less work has explored specialist tertiary units. Undoubtedly such atypical sites have many confounders, including staff make-up and skills, and a cohort of patients whose psychosis is treatmentrefractory. Nevertheless, evaluating the work of centres of proposed excellence is clearly worthwhile. Works by Nirodi et $a l,{ }^{12}$ Ker \& Anderson ${ }^{13}$ and Shepherd et al ${ }^{14}$ describe the difficulties and rationale for tertiary services more broadly, particularly for treatment-resistant depression, and some of these arguments can be equally considered for refractory psychosis. The nature of commissioning and costing of services in the UK and a push towards primary care management of common disorders mitigate against specialist services. Furthermore, a culture of senior clinicians feeling variously that they 'should' know how to manage 'difficult' cases, clinical insecurity or clinical overconfidence might be barriers to obtaining a second opinion. However, tertiary care can be argued to afford three broad advantages. First, 'general' psychiatrists cannot realistically remain experts in all conditions and with the most recent research developments, nor will they necessarily have the multidisciplinary resources to implement them. Second, specialist services can act as an expert resource for consultation and in training generalists, aiding clinical development and confidence. Third, the academic links

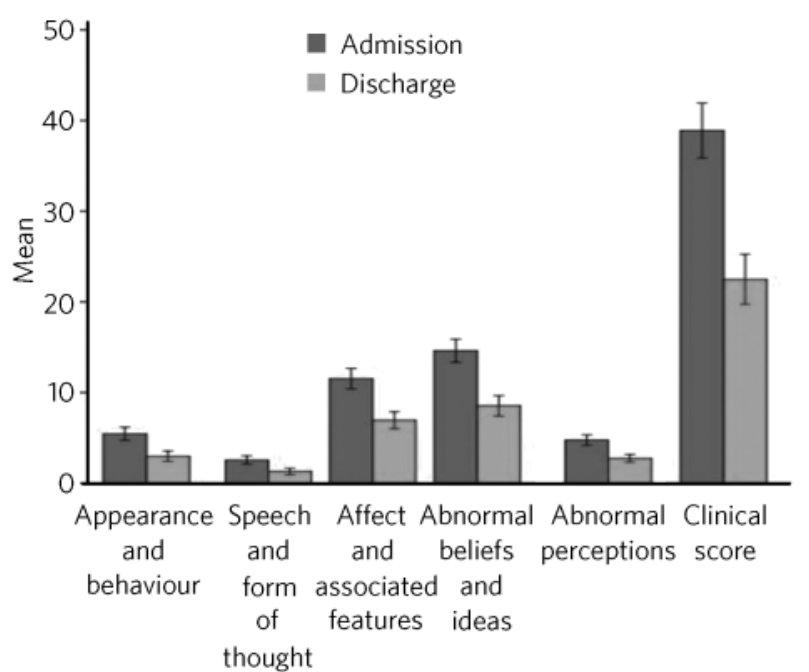

Fig 1 Mean OPCRIT scores from admission to discharge, with 95\% confidence interval error bars. typically found in centres of excellence facilitate evaluation and more rapid integration of novel therapeutic developments.

\section{Study limitations}

There are a few caveats that require highlighting when reviewing a naturalistic data-set with no control group. Primary issues are that a full data-set was not available for all those admitted; comparisons were only possible at two time points in individuals; patients are admitted when chronically unwell and failing to respond to treatment, and may potentially be unlikely to be offered medically recommended discharge until some improvement is shown; adherence to medication may be better in a closely monitored in-patient unit. Nevertheless, there was evidence for a statistically significant improvement, across all clinical domains, in a cohort of patients with psychosis deemed unresponsive to standardised secondary care. Although the OPCRIT is considered a reliable and well-validated tool, its design was primarily for extracting diagnostic information from case notes. Its use in retrospective data collection and as a mechanism for scaling symptom severity is open to challenge ${ }^{15}$ and one cannot exclude the inadvertent biases introduced during the rating process. Two of the authors (S.N.S. and G.D.), both psychiatrists, collected the OPCRIT data: they undertook interrater reliability training, and a test-retest on a random sample of ten sets of notes showed good reliability. The costings model is inevitably somewhat crude, but the factors utilised were those that were identified from earlier cost-effectiveness studies and consistently noted in the patient records. Nevertheless, future work might undertake a more rigorous exploration of pre- and post-treatment costs as well as longer-term follow-up of clinical and financial outcomes over several years. The patient/demographic variables, illness variables (such as duration of illness, number and length of episodes), and clinical input variables (duration of admission, number and nature of treatments trialled) were not explored as data variables. Inevitably with the well-established issue of responder bias, the findings of any survey with low total numbers must be interpreted judiciously.

\section{Challenges to tertiary services}

There are several logistical challenges in provision of specialist services, particularly where these are geographically distant from the patient's home, making the necessary communication with carers and locality more challenging. There are some approaches which can mitigate these to 
some degree; for example, the National Psychosis Unit holds monthly carers' groups with an aim of providing access to support and information and to discuss their lived experiences. In this context, the care programme approach provides an essential framework for regular communication between the locality teams, carers and the specialist centre and, in our experience, this forum is viewed very positively by all participants. Finally, the National Psychosis Unit is unusual in being integrated with the Psychosis Clinical Academic Group at the Institute of Psychiatry, King's College London, enabling very close links with active researchers and access to novel treatments. ${ }^{16-18}$ State-ofthe-art investigations ${ }^{19-21}$ and therapeutic monitoring are easily available, as are the necessary liaison with specialised pharmacy, haematology and cardiology. The recovery-based focus is also facilitated by access to a full range of occupational therapeutic and highly specialised psychological therapies for psychosis. Our initial findings support the effectiveness of admission to a specialist in-patient service, but longer-term prospective data are required, particularly looking at the specific patient and clinical input factors that might affect outcomes. ${ }^{22-24}$

\section{Acknowledgements}

We are grateful to the medical students from King's College London who assisted with some of the data collection: Amber Appleton, Poppy Elmhirst, Laura Bottwood and Sarah Elsabagh. We also thank Dr Ben Robinson for assistance in preparing some of the demographic material.

\section{About the authors}

S. Neil Sarkar is a consultant psychiatrist, Central and North West London NHS Foundation Trust, and researcher, Cognition, Schizophrenia and Imaging Laboratory, Department of Psychosis Studies, Institute of Psychiatry, King's College London; Derek K.Tracy is a researcher, Cognition, Schizophrenia and Imaging Laboratory, and a consultant psychiatrist, Oxleas NHS Foundation Trust; Maria-Jesus Mateos Fernandez is a researcher and Natasza Nalesnik is a PhD student at Cognition, Schizophrenia and Imaging Laboratory; Gurbinder Dhillon is a core trainee, Oxleas NHS Foundation Trust; Juliana Onwumere is a clinical psychologist and Anne-Marye Prins is a core trainee, South London and Maudsley NHS Foundation Trust; Karen Schepman is a researcher, Tracy Collier is a researcher and Thomas $\mathbf{P}$. White is a Post-Doctoral researcher, Cognition, Schizophrenia and Imaging Laboratory; Anita Patel is a health economist, Health Service and Population Research Department, Institute of Psychiatry, King's College London; Fiona Gaughran is a consultant psychiatrist, South London and Maudsley NHS Foundation Trust; Sukhwinder S. Shergill is a reader in psychiatry, Cognition, Schizophrenia and Imaging Laboratory, and a consultant psychiatrist, South London and Maudsley NHS Foundation Trust.

\section{References}

1 Hasan A, Falkai P, Wobrock T, Lieberman J, Glenthoj B, Gattaz WF, et al. World Federation of Societies of Biological Psychiatry (WFSBP) Guidelines for Biological Treatment of Schizophrenia, part 1: update 2012 on the acute treatment of schizophrenia and the management of treatment resistance. World J Biol Psychiatry 2012; 13: 318-78.

2 Tracy DK, Sendt KV, Shergill SS. Antipsychotic polypharmacy: still dirty, but hardly a secret. A systematic review and clinical guide. Curr Psychopharmacol 2013; 2: 91-130.

3 Sendt KV, Giaroli G, Tracy DK. Beyond dopamine: glutamate as a target for future antipsychotics. ISRN Pharmacol 2012; article id 427267.
4 Papanastasiou E, Stone JM, Shergill S. When the drugs don't work: the potential of glutamatergic antipsychotics in schizophrenia. $\mathrm{Br} J$ Psychiatry 2013; 202: 91-3.

5 Barnes TR. Evidence-based guidelines for the pharmacological treatment of schizophrenia: recommendations from the British Association for Psychopharmacology. J Psychopharmacol 2011; 25 : 567-620.

6 Lehman AF, Lieberman JA, Dixon LB, McGlashan TH, Miller AL, Perkins $\mathrm{DO}$, et al. Practice guideline for the treatment of patients with schizophrenia, second edition. Am J Psychiatry 2004; 161 (suppl 2): 1-56.

7 Taylor D, Paton C, Kapur S. Maudsley Prescribing Guidelines. WileyBlackwell, 2012.

8 Howes OD, Vergunst F, Gee S, McGuire P, Kapur S, Taylor D. Adherence to treatment guidelines in clinical practice: study of antipsychotic treatment prior to clozapine initiation. Br J Psychiatry 2012; 201: 481-5.

9 NHS Specialised Services. Definition no. 22 Specialised mental health services (all ages). In Specialised Services National Definitions Set (3rd edn). NHS Specialised Services, 2009.

10 McGuffin P, Farmer A, Harvey I. A polydiagnostic application of operational criteria in studies of psychotic illness. Development and reliability of the OPCRIT system. Arch Gen Psychiatry 1991; 48: 764-70.

11 Patel A, Everitt B, Knapp M, Reeder C, Grant D, Ecker C, et al. Schizophrenia patients with cognitive deficits: factors associated with costs. Schizophr Bull 2006; 32: 776-85.

12 Nirodi P, Mitchell AJ, Mindham RHS. Survey of expert second opinions in a tertiary psychiatric out-patient clinic in the Yorkshire region between 1988 and 2000. Psychiatr Bull 2003; 27: 416-20.

13 Ker S, Anderson I. Service innovations: developing a specialised (tertiary) service for the treatment of affective disorders. Psychiatr Bull 2006; 30: 103-5.

14 Shepherd DJ, Insole LJ, McAllister-Williams RH, Ferrier IN. Are specialised affective disorder services useful? Psychiatr Bull 2009; 33 41-3.

15 Mihalopoulos C, McGorry P, Roberts S, McFarlane C. The procedural validity of retrospective case note diagnosis. Aust N Z J Psychiatry 2000; 34: 154-9.

16 Michalopoulou PG, Azim A, Tracy D, Shergill SS. Ropinirole as an effective adjunctive treatment for clozapine-resistant negative symptoms in simple schizophrenia: a case report. J Clin Psychopharmacol 2012; 32: 719-20.

17 Patel SS, Attard A, Jacobsen P, Shergill S. Acetylcholinesterase inhibitors (AChEl's) for the treatment of visual hallucinations in schizophrenia: a review of the literature. BMC Psychiatry 2010; 10: 69.

18 Patel SS, Attard A, Jacobsen P, Shergill S. Acetylcholinesterase inhibitors (AChEl's) for the treatment of visual hallucinations in schizophrenia: a case report. BMC Psychiatry 2010; 10: 68.

19 Gromann PM, Tracy DK, Giampietro V, Brammer MJ, Krabbendam L, Shergill SS. Examining frontotemporal connectivity and rTMS in healthy controls: implications for auditory hallucinations in schizophrenia. Neuropsychology 2012; 26: 127-32.

20 Simons CJ, Tracy DK, Sanghera KK, O'Daly O, Gileen J, Domingues MD, et al. Functional magnetic resonance imaging of inner speech in schizophrenia. Biol Psychiatry 2010; 67: 232-7.

21 Tracy DK, O'Daly O, Joyce DW, Michalopoulou PG, Basit BB, Dhillon G, et al. An evoked auditory response fMRI study of the effects of rTMS on putative AVH pathways in healthy volunteers. Neuropsychologia 2010; 48: $270-7$.

22 Craddock N, O'Donovan MC, Owen MJ. Phenotypic and genetic complexity of psychosis. Invited commentary on ... Schizophrenia: a common disease caused by multiple rare alleles. Br J Psychiatry 2007; 190: 200-3.

23 Falkenburg J, Tracy DK. Sex and schizophrenia: a review of gender differences. Psychosis 2012; http://www.tandfonline.com/doi/full/ 10.1080/17522439.2012.733405, accessed July 2013

24 Owen MJ, O'Donovan MC, Thapar A, Craddock N. Neurodevelopmental hypothesis of schizophrenia. Br J Psychiatry 2011; 198: 173-5. 